\title{
Evaluating transit-served areas with non-traditional data: An exploratory study of Shenzhen, China
}

\author{
Jiangping Zhou \\ The University of Hong Kong \\ zhoujp@hku.hk
}

\section{Haitao Liu}

The University of Hong Kong

barryliu@connect.hku.hk

\author{
Qihao Wang \\ Lay-out Planning Consultants Co., Ltd \\ wangqh9939@aliyun.com
}

\begin{abstract}
In this study, transit-served areas (TSAs) are defined as areas within a reasonable distance (e.g., 800 meters) of transit services. TSAs have two key dimensions: physical features (e.g., land-use density and mix) and performance (regarding human behaviors). Non-traditional data (NTD) (e.g., social media check-ins and cellular network data) can supplement traditional data (TD) (e.g., interviews and censuses) to enhance studies and monitoring of TSAs. A case study of Shenzhen, China, illustrates how to combine NTD and TD to evaluate the features and performance of 167 TSAs along metro lines. It finds that NTD can be used to formulate new indicators to measure and monitor the two dimensions of TSAs; the features and performance of different TSAs vary significantly; point of interest (POI) efficiency, or the average users attracted by each POI, can be a useful indicator to differentiate TSAs' performance; the POI efficiency of a single TSA can vary across days and the POI efficiency of an extremely efficient or inefficient TSA can be totally different across days; and the combination of NTD and TD can effectively help locate extreme TSAs and explain factors contributing to the extremity.
\end{abstract}

Keywords: Transit-served area, feature, performance, non-traditional data, Shenzhen

\section{Article history:}

Received: August 31, 2017

Received in revised form:

June 8, 2018

Accepted: August 31, 2018

Available online: December 28,

2018

\section{Introduction}

With the rise in personal wealth, many countries have seen increased private car usage and subsequent or correlated negative consequences or phenomena such as traffic congestion, urban sprawl, sedentary lifestyle, obesity prevalence and air pollution (Newman \& Kenworthy, 1999; Frank, Saelens, Powell, \& Chapman, 2007). Making such non-driving-alone modes as transit, walking and biking more convenient and competitive has become a priority of many national/city governments. In China, the national government launched a transit-metropolis program in 2011; later, 34 cities were selected and funded to

Copyright 2018 Jiangping Zhou, Qihao Wang, \& Haitao Liu

http://dx.doi.org/10.5198/jtlu.2018.1296

ISSN: 1938-7849 | Licensed under the Creative Commons Attribution - Noncommercial License 4.0

The Journal of Transport and Land Use is the official journal of the World Society for Transport and Land Use (WSTLUR) and is published and sponsored by the University of Minnesota Center for Transportation Studies. 
pilot various transport-metropolis projects (Zhou, 2016). In Europe, the introduction of newer railway technologies, concurrent or subsequent redevelopment of railway stations and their surroundings have been common across countries in the past three decades or so (Bertolini, Curtis, \& Renee, 2012). In the US, cities like Denver, Twin Cities and Los Angeles have seen planned light rail lines completed sooner than scheduled or lines built on top of the original plan(s) because of residents therein were willing to pay extra taxes to fund transit projects. Conscious, proactive and concerted efforts like the above have enhanced and/or engendered more and more transit-served areas (TSAs), areas that are within a reasonable distance (e.g., 800 meters) of a transit stop.

TSAs should be both a "node" and a "place" for a transit system and even for a city/region (Bertolini, 1996, 1999; Cervero, Guerra, \& Al, 2017). The existence and quality of TSAs are crucial to the success of the transit system and even to the city/region it serves. Having such belief, quite a few authors have defined and studied TSAs. But these authors have used different nomenclatures to define TSAs, for example, "transit-served neighborhoods", "transit-served subcity nodes", "transit served real estate development", "transit-rich neighborhood", "transit-served nodes and corridors", "station area" and "station precinct” (Bertolini et al., 2012, p. 31; Cervero, Ferrell, \& Murphy, 2002, p.75; Cervero et al., 2004, p. S10, S13, 139, 177, 322, 349; Carlton, 2007, p.1; Olaru \& Curtis, 2015, p.6; Renee, 2009, p.3; Wang \& Woo, 2017, p.183).

All TSAs should enjoy certain level of transit services. Assume that the level is sufficient or more than sufficient to serve the demand of (potential) transit riders, then we can compare TSAs to a coin with two sides. One side is about all the desired features, especially such physical features as the degree or quality of transit access, mixed land use, pedestrian-friendly design and development density (Dill, 2008). Most of the TSA features (TSAF) require a large amount of resources and efforts to be achieved. The other side of TSAs is such human behaviors as residential choice, activity location, transit usage and walking, which have significant impacts on energy consumption, the environment, society and even public health (Calthorpe 1993; Cervero, 1998; Morris \& Hardman, 1997). Decision-makers as well as the public normally expect that extra or enhanced TSAF because of public investment engender better behaviors/outcomes (hereafter, we call these "TSA performance--TSAP"). Given the variation in TSAF and/or TSAP, there are different categories of TSAs, e.g., transit-oriented developments (TODs), i.e., high-performance TSAs, transit-adjacent developments (TADs), which only have some features or achieve partial performance of a successful TOD, and low-performing TSAs, where development density are so low that they do not even generate a decent amount of transit ridership (c.f., Cervero et al., 2004, 2017; Dill, 2008; Hale, 2014; Olaru \& Curtis, 2015; Renne, 2009).

In such primate cities as Shanghai, Tokyo and New York where transit supply is relatively abundant and transit usage is continuously popular, millions of transit riders have already lived/worked in TSAs. Some called those cities "city of rail" (Liu, Du, \& Zhao, 2013). In such emerging cities as Shenzhen, decision-makers are hoping to build a "city on the rail" as well, that is, putting most residents/workers in TSAs and enticing more and more to use rail transit (DT Caijing, 2017). Quantifying/Assessing TSAP and TSAF and examining relationships between them would therefore be of importance to decisionmakers. Those efforts would allow them to effectively monitor and guide TSAF changes, which in turn help them improve TSAP more proactively and consciously (c.f., Singh, Fard, Zuidgeest, Brussel, \& van Maarseveen, 2014; Singh, Lukman, Flacke, Zuidgeest, \& van Maarseveen, 2017; TCRP, 2007).

TODs, narrowly defined as a subset of TSAs in this study, have been intensively studied for decades. This has produced much knowledge such as desired features of a TOD and how to achieve a performing/successful TOD (Caltrans, 2002; Cervero et al., 2002; Cervero et al. 2004; Cervero et al., 2017; ITDP, 2017; Jeihani \& Zhang, 2013; TCRP, 2002, 2007). TSAs other than TODs, however, have been comparatively overlooked. Research gaps exist in topics on non-TOD TSAs, their feature/ 
performance measurement, changes, and related mechanisms. This is partially because existing TOD/ TSA studies rely so heavily and even solely on traditional data (See for instance, Table 1 below). But it is costly and time-consuming to collect and update these data for a large number of TODs or TSAs frequently. The emergence of non-traditional data (NTD) (e.g., smartcard swipes, on-line maps and social media check-ins) can enhance and supplement traditional data. NTD is automatically and continuously collected and updated. They contain information over more time spans as well as across much more TSAs than traditional data. NTD like the Oyster Card data in London, for instance, can be used to quantify passenger flows at all the 296 subway stations in the city over more time intervals than ever before, providing us with clearer and more continuous pictures of station hubs and the station usage (Batty, 2013; Reades, Zhong, Manley, Milton, \& Batty, 2016). In addition, such NTD as OpenStreetMap (OSM) and Google Route Planner can be used to efficiently quantify intersection density, average block size and metro services frequency (Lyu, Bertonlini, \& Pfeffer, 2016). In short, when NTD is introduced into TSA studies, TSAF and TSAP can be measured more efficiently and frequently than ever before. Learning from Batty (2013), Reades et al. (2016) and Lyu et al. (2016), we could, for instance, continuously quantify and measure the performance and features of all the TSAs in a city purely based on smartcard data and OSM. This would enhance and supplement existing studies or monitoring of TSAs based on such traditional data as on-board surveys and land-use maps, frequent updates of which are time-consuming and labor-intensive.

The purposes of this study are three folds. First, it proposes a new taxonomy of TSAs, showing that TODs have been more extensively studied before but there still exists a research gap regarding TSAs that are not TODs. Second, it revisits existing studies on TSAs by paying special attention to (a) TSAF and TSAP indicators people formulated to categorize and evaluate TSAs; (b) input data people used to compute those indicators and how NTD can supplement and even substitute the traditional data in the computation of those indicators. Third, it presents an exploratory study to illustrate (a) how NTD can supplement and even substitute the traditional data in the computation of the TSAF and TSAP indicators; (b) how the proposed taxonomy can be used to classify and evaluate a large number of TSAs, regardless of they are a TOD or not.

The remaining of this manuscript is organized as follows. Section 2 (the next section) describes the proposed new taxonomy. Section 3 revisits existing studies on various TSAs. Section 4 presents a case study to show how NTD, alone, or its combination with traditional data can be used to evaluate TSAF and TSAP and to understand variances in the performance across the (extreme) TSAs in Shenzhen, China. Section 5 concludes and discusses future research directions.

\section{A new taxonomy of TSAs}

In the past few decades, the transit-land use integration (TLUI) has received an increased attention among scholars and policy-makers (Loo, Chen, \& Chan, 2010; Loo \& du Verle, 2017; Olaru \& Curtis, 2015; Suzuki, Cervero, \& Luchi, 2013; TCRP, 2002, 2007). TLUI has engendered and/or enhanced more and more TSAs, TODs in particular. Besides providing people with good transit services, performing TODs share at least the following (physical) features:

- Mixed-use development;

- Development that is close to and well served by transit;

- Development that is conductive to transit riding;

- Pedestrian friendliness;

- High transit use;

- High residential and business density;

- Presence of quality cycling and walking facilities (TCRP, 2002; Dill, 2008; Singh et al., 2017). 
Not all TSAs can have the above features. In the US, for instance, many cities or cities' subdivisions do not even have any successful TODs (Cervero et al., 2004). In cities known for TODs, e.g., Portland, Oregon, not all TSAs have the agreed-on features of TODs either (Dill, 2008; Cervero et al., 2017). Thus, there is a wide variety of TSAs but TODs are often only a small subset. If we can somehow quantify TSAF/TSAP and establish some cut-off point based on the quantification, we can have different categories (subsets) of TSAs. Figure 1 depicts a new taxonomy of TSAs and non-TSAs, which are measured in two dimensions: one is about the (physical) features (X-Axis) and the other is about the performance (Y-Axis). The origin, where the X-Axis and Y-Axis intersect, is the cut-off point, which can be, for instance, the average ratio of transit usage in a neighborhood in a region (for performance) and the average distance to the nearest transit station among residents in the region (for features). Non-TSAs will be those areas outside a threshold distance (e.g., 800 meters) of any transit stops. Based on this taxonomy, we can then have two categories of non-TSAs (performing vs. underperforming) and TSAs (performing vs. underperforming), respectively.

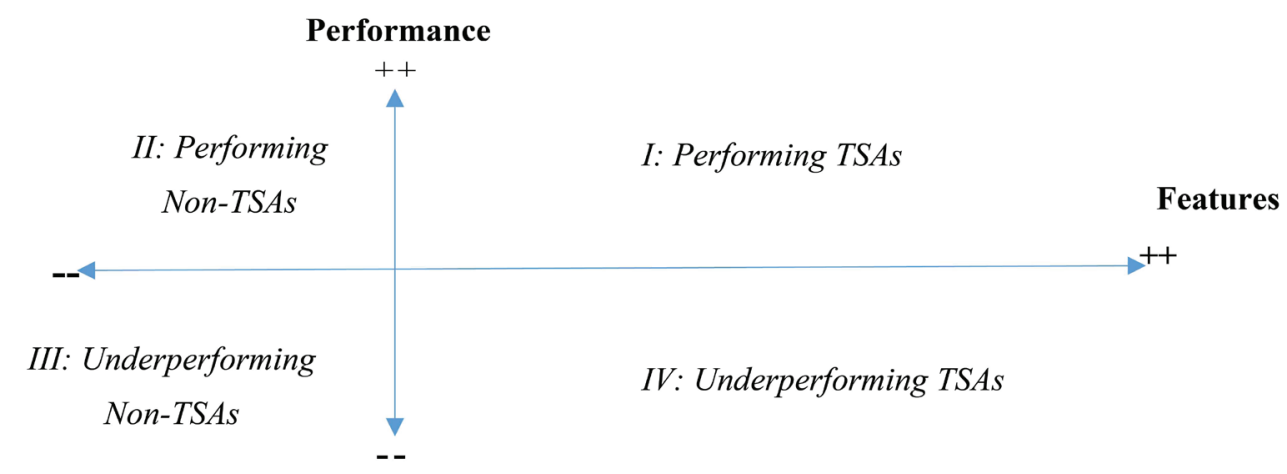

Figure 1: A proposed taxonomy of TSAs and non-TSAs

Similarly, we can further categorize TSAs into four quadrants (groups) using some extra cut-off point. In that case, there would also be four types of TSAs in Quadrants I to IV (see Figures 6 and 7 for example).

\section{$3 \quad$ Related literature}

Of various TSAs, TODs have received by far the most attention in existing studies. This may not be surprising as TODs are the most desirable TSAs, which have different kinds of social, environmental and economic benefits (e.g., see Calthorpe, 1993; Cervero \& Kockelman, 1997; Cervero et al., 2002; Cervero et al., 2004; Cervero et al., 2017; Dittmar \& Ohland, 2004; TCRP, 2007). Existing studies have generated rich (but somehow biased) knowledge about TSAs such as:

- How different authors define or delineate TODs (e.g., Boarnet \& Crane, 1998; Calthorpe, 1993; Cervero et al., 2004; Renne, Bartholomew, \& Wontor, 2011);

- What characteristics a successful TOD should have and how to quantify and evaluate the efficacy of these characteristics (e.g., Bernick \& Cervero, 1997; Bukowaski, Boatman, Ramierz, \& Du, 2013; Cervero et al., 2004; Cervero \& Kockelmam, 1997; Hale, 2014; Lyu et al., 2016; Renee, 2009; Singh et al., 2017); 
- How to finance and implement TODs (e.g., Cervero, 1998; Cervero et al., 2004; Belzer \& Autler, 2002; Cervero \& Murakami, 2009);

- How TODs can affect travel behaviors, residents' housing choice and/or businesses (e.g., Cervero et al., 2004; Dill, 2008; Dong, 2017; Griffiths \& Curtis, 2017; Jeihani \& Zhang, 2013; Kahn, 1997; Kamruzzaman, Shatu, Hine, \& Turrell, 2015; Ong, Pech, \& Ray, 2014).

Existing studies, however, have comparatively overlooked the following topics:

(a) A taxonomy of all TSAs based on some predefined baselines about the TSAF and TSAP, as described above and in Figure 1;

(b) Non-TOD TSAs and how to measure their performance relative to some baseline(s);

(c) Spontaneous or government-led changes of all TSAs and related mechanisms, especially the devolution of TSAs and incremental market-based improvements of TSAs, where TSAs do not become TODs but perform better than before as compared to some predefined baselines (e.g., the mean regional population density or the average rate of transit usage across local TSAs);

(d) How to continuously measure, monitor and improve TSAP across different categories of TSAs based on NTD or its combination with traditional data.

In this study, we focus on (a) and (d). We describe an exploratory case study regarding how we classify TSAs in Shenzhen, China based on the proposed taxonomy and by adopting NTD. We also illustrate how to combine NTD and traditional data to understand better factors contributing to the performance of four representative TSAs.

\subsection{TSAF and TSAP indicators}

Following the above proposed taxonomy of TSAs (see Figure 1), we can reclassify and review existing studies on TSAs and better elaborate the research gaps therein. Table 1 presents a synopsis of our findings. To increase the readability of the findings and at some risk of overlooking some delicacies and complexities in TSAF, we used the 5 D's (i.e., Density, Design, Destination, Distance and Diversity) by Ewing and Cervero (2001) to synthesize the TSAF whenever feasible. There have been more recent studies on D's since Ewing and Cervero (2001). At some risk of oversimplifying the complexity of D's, we still categorize the former based on the 5 D's. It should be noted that such authors as Cervero et al., (2017), ITDP (2017), Lyu et al. (2016) and Singh et al. (2017) have synthesized and recommended more TSAF and/or TSAP indicators than the 5 D's. According to our best knowledge of the existing studies, we interpret the 5 D's as follows.

Density (D1): building/dwelling unit density as well as density of facilities such as sidewalks and retail shops.

Design (D2): facilities and the built environment are of human scale, attractive and pedestrian friendly.

Destination (D3): there are facilities such as schools, offices and shops that of interest to local residents/transit riders. Preferably, all these facilities are within a walking distance of a transit stop.

Distance (D4): accessibility to meaningful destinations such as shops, public spaces and transit stops.

Diversity (D5): mixed land use and diverse building types/ages.

For TSAP, there has not been something concise like the 5 D's to characterize it. Thus, we must invent our own: "CUPO". This expression was created after we reviewed the literature between 1993 and 2017 that we collected through such popular refereed paper databases as Web of Social Sciences, Google Scholar and ProQuest. Meaning of each letter in CUPO is given as follows.

"C": car usage/dependence and corresponding negative externalities (in particular, traffic congestion, vehicle miles traveled (VMT) and polluted emissions); 
"U": usage and positive externalities of alternative modes to driving alone (in particular, transit, biking and walking);

"P": place enhancement and creation (e.g., a more walkable neighborhood);

"O": overall economic social and environmental benefits (e.g., local business gains, reduced outlays for roads, improved safety for pedestrians and cyclist and more open spaces).

Table 1: Existing studies of TSAs from a new perspective

\begin{tabular}{|c|c|c|c|c|c|c|}
\hline Source & $\begin{array}{l}\text { TSA } \\
\text { category }\end{array}$ & $\begin{array}{l}\text { Features } \\
\text { Considered/ } \\
\text { Proposed }\end{array}$ & $\begin{array}{l}\text { Performance } \\
\text { Considered/ } \\
\text { Proposed }\end{array}$ & Input data & $\begin{array}{l}\text { Feature Measure- } \\
\text { ment/ Indicator }\end{array}$ & $\begin{array}{l}\text { Performance } \\
\text { Measurement/ } \\
\text { Indicator }\end{array}$ \\
\hline $\begin{array}{l}\text { Calthorpe, } \\
1993\end{array}$ & TOD & D1-4 & $\mathrm{C} ; \mathrm{P} ; \mathrm{O}$ & $\begin{array}{l}\text { Not men- } \\
\text { tion (N.M.) }\end{array}$ & $\begin{array}{l}\text { Walking distance } \\
\text { to meaningful des- } \\
\text { tinations and other } \\
\text { narratives }\end{array}$ & $\begin{array}{l}\text { Car ownership; } \\
\text { Auto trips; Traffic } \\
\text { congestion; Hous- } \\
\text { ing cost; Energy } \\
\text { used; Air pollution; } \\
\text { VMT }\end{array}$ \\
\hline $\begin{array}{l}\text { Cervero and } \\
\text { Kockelman, } \\
1997\end{array}$ & TSA & $\mathrm{D} 1,2,5$ & $\mathrm{C} ; \mathrm{U}$ & $\begin{array}{l}\text { Travel diary; } \\
\text { Land-use re- } \\
\text { cords; Field } \\
\text { surveys: } \\
\text { Literature } \\
\text { review }\end{array}$ & $\begin{array}{l}\text { Accessibility to jobs; } \\
\text { Dissimilarity index; } \\
\text { Entropy; Vertical } \\
\text { mixture of land } \\
\text { uses; Intensity of } \\
\text { land uses; Activ- } \\
\text { ity center mixture; } \\
\text { Commercial inten- } \\
\text { sities; Proximity to } \\
\text { commercial-retail } \\
\text { uses; Street patterns; } \\
\text { Ped./Bike facility } \\
\text { provision; Site de- } \\
\text { sign (esp. parking) }\end{array}$ & $\begin{array}{l}\text { Mode share; VMT; } \\
\text { Trip rates; Popula- } \\
\text { tion/ Employment } \\
\text { density* }^{*}\end{array}$ \\
\hline $\begin{array}{l}\text { Bernick and } \\
\text { Cervero, } \\
1997\end{array}$ & $\begin{array}{l}\text { TSA } \\
\text { ("Transit } \\
\text { village") }\end{array}$ & $\mathrm{D} 1,2,5$ & $\mathrm{C} ; \mathrm{U}$ & & $\begin{array}{l}\text { 10-15 dwell units } \\
\text { per acre; Five-min's } \\
\text { walk to many des- } \\
\text { tinations; Grid-like } \\
\text { streets }\end{array}$ & $\begin{array}{l}\text { Non-driving-alone } \\
\text { trips }\end{array}$ \\
\hline $\begin{array}{l}\text { Cervero et al., } \\
2004\end{array}$ & TOD & D1,2,5; & C; U; P; O & & $\begin{array}{l}\text { 20-30 dwell units } \\
\text { per acre and FARs } \\
\geq 1 \text { for urban TODs } \\
\text { and other narratives }\end{array}$ & $\begin{array}{l}\text { Transit rider- } \\
\text { ship and associ- } \\
\text { ated revenue gains; } \\
\text { Congestion relief; } \\
\text { Land conservation; } \\
\text { Reduced outlays for } \\
\text { roads and improved } \\
\text { road safety }\end{array}$ \\
\hline
\end{tabular}




\begin{tabular}{|c|c|c|c|c|c|c|}
\hline $\begin{array}{l}\text { Boarnet and } \\
\text { Crane, } 1998\end{array}$ & TOD & $\mathrm{D} 1,2$ & U; O & $\begin{array}{l}\text { Zoning data } \\
\text { near stations }\end{array}$ & $\begin{array}{l}\text { Land uses around } \\
\text { stations }\end{array}$ & $\begin{array}{l}\text { Supply of transit- } \\
\text { based housing and } \\
\text { its financial viability } \\
\text { (Also mentioned } \\
\text { others' measure- } \\
\text { ments such as more } \\
\text { transit trips and less } \\
\text { air pollution) }\end{array}$ \\
\hline $\begin{array}{l}\text { Arrington } \\
\text { and Parker, } \\
2001\end{array}$ & TOD & $\mathrm{D} 1,2,4,5$ & C; U; P; O & N.M. & NA & NA \\
\hline $\begin{array}{l}\text { Belzer and } \\
\text { Autler, } 2002\end{array}$ & TOD & D3-5 & C; U; O & N.M. & NA & NA \\
\hline $\begin{array}{l}\text { Cervero et al., } \\
2002\end{array}$ & $\begin{array}{l}\text { TAD; } \\
\text { TOD }\end{array}$ & $\begin{array}{l}\text { D1,2,4,5; } \\
\text { Other } \\
\text { non-physical } \\
\text { matters such } \\
\text { as cultural } \\
\text { history, social } \\
\text { and human } \\
\text { capital } \\
\end{array}$ & U; O & $\begin{array}{l}\text { Secondary } \\
\text { sources }\end{array}$ & $\begin{array}{l}\text { Compactness; } \\
\text { Mixed use; Transit } \\
\text { connectivity; Site } \\
\text { design }\end{array}$ & $\begin{array}{l}\text { Transit patronage; } \\
\text { Rent premium }\end{array}$ \\
\hline $\begin{array}{l}\text { Caltrans } \\
(2002)\end{array}$ & TOD & $\mathrm{D} 1,2,4,5$ & U; O. & N.M. & $\begin{array}{l}\text { Development } \\
\text { Density; Walk time } \\
\text { to transit }\end{array}$ & $\begin{array}{l}\text { Transit use; Sprawl } \\
\text { trend; Safety; } \\
\text { VMT; Disposable } \\
\text { household income; } \\
\text { Air pollution; En- } \\
\text { ergy consumption; } \\
\text { Infrastructure costs; } \\
\text { Affordable housing }\end{array}$ \\
\hline $\begin{array}{l}\text { Dittmar and } \\
\text { Ohland, } \\
2004\end{array}$ & $\begin{array}{l}\text { TAD; } \\
\text { TOD }\end{array}$ & $\begin{array}{l}\mathrm{D} 1,2,5 ; \\
\text { Location- } \\
\text { efficient de- } \\
\text { velop-ment; } \\
\text { Convenient } \\
\text { transit } \\
\text { services }\end{array}$ & $\mathrm{O}$ & N.M. & $\begin{array}{l}\text { Only some narra- } \\
\text { tives (See., p.7) }\end{array}$ & $\begin{array}{l}\text { Overall: Improved } \\
\text { quality of life; } \\
\text { Healthy metro } \\
\text { economies }\end{array}$ \\
\hline
\end{tabular}




\begin{tabular}{|c|c|c|c|c|c|c|}
\hline $\begin{array}{l}\text { NCHRP, } \\
2005\end{array}$ & TOD & NA & C; U; P; O & N.M. & N.M. & $\begin{array}{l}\text { Vary depending on } \\
\text { survey responses. } \\
\text { Top } 10 \text { recom- } \\
\text { mended: Transit } \\
\text { ridership; Density- } \\
\text { population/hous- } \\
\text { ing; Quality of } \\
\text { streetscape design; } \\
\text { Ped. activity/safety; } \\
\text { Public perception; } \\
\text { Mode connections; } \\
\text { Parking configura- } \\
\text { tion }\end{array}$ \\
\hline Renee, 2007 & TSA & $\begin{array}{l}\text { D1,2,4,5; } \\
\text { Parking }\end{array}$ & C; U; P; O & $\begin{array}{l}\text { Official data } \\
\text { plus ad-hoc } \\
\text { surveys }\end{array}$ & $\begin{array}{l}\text { Population/ } \\
\text { Housing density; } \\
\text { Street quality; } \\
\text { Public space; } \\
\text { Land cover/ } \\
\text { use; Pedestrian } \\
\text { accessibility; } \\
\text { Parking inventory }\end{array}$ & $\begin{array}{l}5 \text { categories, } 21 \\
\text { indicators, ranging } \\
\text { from travel behav- } \\
\text { iors to the policy } \\
\text { context }\end{array}$ \\
\hline TCRP, 2007 & TOD & \begin{tabular}{|l|} 
D1,2,4; \\
Managed \\
parking \\
supply
\end{tabular} & C; U; O & $\begin{array}{l}\text { Travel } \\
\text { surveys; } \\
\text { Rider count; } \\
\text { Building } \\
\text { data }\end{array}$ & $\begin{array}{l}\text { "TOD-index" } \\
\text { reflecting distance } \\
\text { to transit, walkabil- } \\
\text { ity, transit services, } \\
\text { land-use mix, den- } \\
\text { sity and parking }\end{array}$ & $\begin{array}{l}\text { Primary transit } \\
\text { mode; Car owner- } \\
\text { ship; VMT; Level } \\
\text { of congestion }\end{array}$ \\
\hline Kahn, 2007 & TOD & D4 & $\mathrm{O}$ & Census data & $\begin{array}{l}\text { Proximity to rail } \\
\text { transit }\end{array}$ & $\begin{array}{l}\text { Average housing } \\
\text { prices; Ratio of } \\
\text { college graduates in } \\
\text { a census track }\end{array}$ \\
\hline Dill, 2008 & TSA & $\mathrm{D} 1,2,4,5$ & $\mathrm{U}$ & Surveys & $\begin{array}{l}\text { Distance to transit; } \\
\text { Land-use mix; Pe- } \\
\text { destrian friendliness }\end{array}$ & Transit use \\
\hline Renee, 2009 & $\begin{array}{l}\text { TAD; } \\
\text { TOD }\end{array}$ & $\mathrm{D} 1,2,5$ & $\mathrm{C} ; \mathrm{U} ; \mathrm{O}$ & $\begin{array}{l}\text { Census } \\
\text { and TOD } \\
\text { databases }\end{array}$ & $\begin{array}{l}\text { Housing density; } \\
\text { Number of street } \\
\text { links; number of } \\
\text { nodes; Typical block } \\
\text { dimensions; Sta- } \\
\text { tion design rating; } \\
\text { Pedestrian/ Bike } \\
\text { accessibility rating }\end{array}$ & $\begin{array}{l}\text { Transit ridership; } \\
\text { Mode share }\end{array}$ \\
\hline
\end{tabular}




\begin{tabular}{|c|c|c|c|c|c|c|}
\hline Loo, 2009 & TOD & $\begin{array}{l}\text { D1,2,4,5; } \\
\text { Parking avail- } \\
\text { ability; Public } \\
\text { transport } \\
\text { services; }\end{array}$ & \multirow[t]{2}{*}{$\mathrm{U}$} & N.M. & $\mathrm{NA}$ & $\mathrm{NA}$ \\
\hline $\begin{array}{l}\text { Loo et al., } \\
2010\end{array}$ & TOD & $\mathrm{D} 2,5$ & & $\begin{array}{l}\text { Transit } \\
\text { companies' } \\
\text { data }\end{array}$ & $\begin{array}{l}\text { Total commercial/ } \\
\text { residential floor } \\
\text { area; Parking area; } \\
\text { Location of the } \\
\text { station (dummy); } \\
\text { Mixed land use } \\
\text { (dummy); General- } \\
\text { ized travel cost to } \\
\text { Midtown; Major } \\
\text { interchange station } \\
\text { (dummy); Number } \\
\text { of bus stops }\end{array}$ & Rail ridership \\
\hline $\begin{array}{l}\text { Sung and Oh, } \\
2011\end{array}$ & $\mathrm{TSA}^{* * *}$ & $\begin{array}{l}\text { D1,4,5; Level } \\
\text { of transit } \\
\text { services }\end{array}$ & $\mathrm{U}$ & $\begin{array}{l}\text { Local transit } \\
\text { plan; Local } \\
\text { transit } \\
\text { service data; } \\
\text { Official land } \\
\text { use and } \\
\text { building } \\
\text { design data; } \\
\text { Smartcard } \\
\text { data** }\end{array}$ & $\begin{array}{l}\text { Number of bus } \\
\text { routes; Average } \\
\text { headway; Distance } \\
\text { between station; } \\
\text { Residential/ Com- } \\
\text { mercial density; } \\
\text { Commercial/ Busi- } \\
\text { ness mix index; } \\
\text { Total road length; } \\
\text { Average road width; } \\
\text { Four-way intersec- } \\
\text { tion density; Dead } \\
\text { end road; Average } \\
\text { building area }\end{array}$ & Transit ridership \\
\hline Curtis, 2012 & TSA $^{* * *}$ & $\mathrm{D} 1,4,5$ & $\mathrm{O}$ & $\begin{array}{l}\text { Official } \\
\text { statistics }\end{array}$ & $\begin{array}{l}\text { Housing; Land } \\
\text { uses }\end{array}$ & $\begin{array}{l}\text { Transit trips; Em- } \\
\text { ployment density* }\end{array}$ \\
\hline $\begin{array}{l}\text { Ratner and } \\
\text { Goetz, } 2013\end{array}$ & TSA $^{* * *}$ & D4 & $\mathrm{O}$ & $\begin{array}{l}\text { Local official } \\
\text { data }\end{array}$ & $\begin{array}{l}\text { Access to a real or } \\
\text { proposed transit } \\
\text { station (dummy) }\end{array}$ & $\begin{array}{l}\text { Dwelling units; } \\
\text { Retail space; Office } \\
\text { space; Medical } \\
\text { space }\end{array}$ \\
\hline $\begin{array}{l}\text { Jeihani and } \\
\text { Zhang, } 2013\end{array}$ & TOD & D1,2,4,5 & $C ; U$ & $\begin{array}{l}\text { Household } \\
\text { travel sur- }\end{array}$ & $\begin{array}{l}\text { Households in } \\
\text { TOD; Household }\end{array}$ & $\begin{array}{l}\text { Transit ridership; } \\
\text { VMT }\end{array}$ \\
\hline $\begin{array}{l}\text { Nasri and } \\
\text { Zhang, } 2014\end{array}$ & & & & $\begin{array}{l}\text { veys; GIS } \\
\text { shapefiles of } \\
\text { TAZs, cen- } \\
\text { sus blocks } \\
\text { and stations }\end{array}$ & $\begin{array}{l}\text { in a rail-accessible } \\
\text { zone; Bus stop } \\
\text { density; Residen- } \\
\text { tial/ Employment } \\
\text { Density; Distance } \\
\text { from CBD; Average } \\
\text { block size }\end{array}$ & \\
\hline
\end{tabular}




\begin{tabular}{|c|c|c|c|c|c|c|}
\hline $\begin{array}{l}\text { Singh et al., } \\
2014\end{array}$ & $\mathrm{TSA}^{* * *}$ & $\mathrm{D} 1,2,4,5$ & NA & $\begin{array}{l}\text { Secondary } \\
\text { spatial and } \\
\text { statistical } \\
\text { data }\end{array}$ & $\begin{array}{l}\text { TOD index } \\
\text { (reflecting density, } \\
\text { diversity, design } \\
\text { and current level of } \\
\text { economic develop- } \\
\text { ment); Transit } \\
\text { connectivity }\end{array}$ & NA \\
\hline $\begin{array}{l}\text { Papa and Ber- } \\
\text { tolini, } 2015\end{array}$ & TSA $^{* * *}$ & D4 & $\mathrm{O}$ & $\begin{array}{l}\text { Official } \\
\text { statistics; } \\
\text { OpenStreet- } \\
\text { Map** }\end{array}$ & $\begin{array}{l}\text { Rail-based acces- } \\
\text { sibility, a condi- } \\
\text { tion for rail use } \\
\text { and an enabler of } \\
\text { travel choices and } \\
\text { behaviors }\end{array}$ & $\begin{array}{l}\text { "TOD degree": } \\
\text { Correlation be- } \\
\text { tween railway net- } \\
\text { work connectivity } \\
\text { and the distribution } \\
\text { of density }\end{array}$ \\
\hline $\begin{array}{l}\text { Norland, } \\
\text { Ozbay, } \\
\text { DiPetrillo, \& } \\
\text { Lyer, } 2014\end{array}$ & TSA & $\mathrm{D} 1,2$ & $\mathrm{C} ; \mathrm{U} ; \mathrm{P} ; \mathrm{O}$ & $\begin{array}{l}\text { Interviews; } \\
\text { Focus } \\
\text { groups and } \\
\text { surveys }\end{array}$ & $\begin{array}{l}\text { Presence of a train } \\
\text { station and redevel- } \\
\text { opments }\end{array}$ & $\begin{array}{l}\text { Frequency of } \\
\text { walking, driving } \\
\text { and using transit; } \\
\text { Potential health } \\
\text { benefits due to } \\
\text { transit proximity; } \\
\text { Cost of travel; } \\
\text { Property value; } \\
\text { Train usage and } \\
\text { highway congestion }\end{array}$ \\
\hline $\begin{array}{l}\text { Noland and } \\
\text { DiPetrillo, } \\
2015\end{array}$ & $\mathrm{TSA}^{* * *}$ & D4 & $\mathrm{U}$ & $\begin{array}{l}\text { Household } \\
\text { survey }\end{array}$ & $\begin{array}{l}\text { Access to transit; } \\
\text { Local street density } \\
\text { and other subjective } \\
\text { evaluation of built } \\
\text { environment }\end{array}$ & $\begin{array}{l}\text { Frequency of alter- } \\
\text { native modes }\end{array}$ \\
\hline $\begin{array}{l}\text { Lyu et al., } \\
2016\end{array}$ & TSA & $\begin{array}{l}\text { D1-5; Park- } \\
\text { ing for cars } \\
\text { and bicycles; } \\
\text { Transit ser- } \\
\text { vices }\end{array}$ & $\mathrm{C} ; \mathrm{U} ; \mathrm{P} ; \mathrm{O}$ & $\begin{array}{l}\text { On-line } \\
\text { open data**; } \\
\text { Census data }\end{array}$ & \multicolumn{2}{|c|}{$\begin{array}{l}\text { Six indicators for "transit", "development" } \\
\text { and "oriented", respectively (not differenti- } \\
\text { ate TSAF and TSAP); Those indicators } \\
\text { can be further condensed and customized } \\
\text { for different cities (e.g., Beijing) }\end{array}$} \\
\hline $\begin{array}{l}\text { Loo and du } \\
\text { Verle, } 2017\end{array}$ & TOD & $\mathrm{D} 1,2,5$ & $\mathrm{U}$ & $\begin{array}{l}\text { Travel } \\
\text { surveys; } \\
\text { Railway } \\
\text { map }\end{array}$ & $\begin{array}{l}\text { Metro/Bus access; } \\
\text { Gross floor areas of } \\
\text { different land uses; } \\
\text { Simpson index of } \\
\text { land uses; Road } \\
\text { length/total area; } \\
\text { Share of built open } \\
\text { space; Total number } \\
\text { of station exists; } \\
\text { Presence of covered } \\
\text { walkway; Number } \\
\text { of retail shops }\end{array}$ & $\begin{array}{l}\text { Mode share; Popu- } \\
\text { lation/Employment } \\
\text { density* }\end{array}$ \\
\hline
\end{tabular}




\begin{tabular}{|c|c|c|c|c|c|c|}
\hline $\begin{array}{l}\text { Cervero, et } \\
\text { al., } 2017\end{array}$ & TOD & $\begin{array}{l}\text { D1-5; Transit } \\
\text { service } \\
\text { frequency }\end{array}$ & $\mathrm{C} ; \mathrm{P} ; \mathrm{O}$ & N.M. & $\begin{array}{l}\text { Density of residents } \\
\text { and employees; } \\
\text { Average block size; } \\
\text { Availability and } \\
\text { quality of urban } \\
\text { living infrastruc- } \\
\text { ture; Access to } \\
\text { and connectivity } \\
\text { of bikeways and } \\
\text { sidewalks; Transit } \\
\text { service frequency; } \\
\text { Place identity (Nar- } \\
\text { ratives only) }\end{array}$ & $\begin{array}{l}\text { VMT; Travel time/ } \\
\text { cost saving; In- } \\
\text { migration }\end{array}$ \\
\hline $\begin{array}{l}\text { Griffiths and } \\
\text { Curtis, } 2017\end{array}$ & TOD & D1,2,4,5 & U & Survey data & $\begin{array}{l}\text { Density of dwelling } \\
\text { units }\end{array}$ & Car trips \\
\hline ITDP, 2017 & TOD & D1-5 & $\mathrm{U} ; \mathrm{O}$ & N.M. & $\begin{array}{l}13 \text { indicators about } \\
\text { accessibility to des- } \\
\text { tinations, diversity, } \\
\text { transit options, den- } \\
\text { sity and percentage } \\
\text { of land dedicated to } \\
\text { motor vehicle }\end{array}$ & N.M. \\
\hline $\begin{array}{l}\text { Singh et al., } \\
2017\end{array}$ & $\mathrm{TSA}^{* * *}$ & $\begin{array}{l}\text { D1,2,4,5; } \\
\text { Parking }\end{array}$ & $\mathrm{O}$ & $\begin{array}{l}\text { Spatial/ } \\
\text { statistical } \\
\text { data from } \\
\text { the govern- } \\
\text { ment or GIS } \\
\text { vendors }\end{array}$ & $\begin{array}{l}\text { Population/ Com- } \\
\text { mercial density; } \\
\text { Entropy; Mixed- } \\
\text { ness; Total length } \\
\text { of walkable/cyclable } \\
\text { paths; Impedance } \\
\text { pedestrian catch- } \\
\text { ment area;User- } \\
\text { friendliness of tran- } \\
\text { sit system; Access } \\
\text { and accessibility; } \\
\text { Parking at station }\end{array}$ & $\begin{array}{l}\text { Capacity utiliza- } \\
\text { tion of transit; } \\
\text { Density of business } \\
\text { establishment }{ }^{* * * * ;} \\
\text { Employment } \\
\text { level**** }\end{array}$ \\
\hline Renee, 2018 & $\mathrm{TSA}^{* * *}$ & $\mathrm{D} 1,2,4$ & $\mathrm{U} ; \mathrm{O}$ & $\begin{array}{l}\text { A national } \\
\text { TOD data- } \\
\text { base }\end{array}$ & Walkability & $\begin{array}{l}\text { Job density*; } \\
\text { Percentage of pro- } \\
\text { fessional jobs*; } \\
\text { Mode share; Car } \\
\text { ownership }\end{array}$ \\
\hline $\begin{array}{l}\text { Rogriguez } \\
\text { and Vergel- } \\
\text { Tovar, } 2017\end{array}$ & TSA & D1-5 & U & $\begin{array}{l}\text { Self-collect- } \\
\text { ed primary } \\
\text { data and } \\
\text { secondary } \\
\text { data from } \\
\text { local transit } \\
\text { agencies }\end{array}$ & $\begin{array}{l}38 \text { variables, rang- } \\
\text { ing from facility } \\
\text { density to land-use } \\
\text { mix }\end{array}$ & $\begin{array}{l}\text { Ridership; Pedes- } \\
\text { trian activities\#; } \\
\text { Land values\#; } \\
\text { Affordability\# }\end{array}$ \\
\hline
\end{tabular}




\begin{tabular}{|l|l|l|l|l|l|l|}
\hline $\begin{array}{l}\text { Zhao and Li, } \\
2018\end{array}$ & TSA** & D2,5 & U; O & Survey data & Land uses & $\begin{array}{l}\text { Patrons of facili- } \\
\text { ties/ shops; Mode } \\
\text { choice;Car owner- } \\
\text { ship }\end{array}$ \\
\hline
\end{tabular}

*Authors tend to treat it as a TSAF influences TSAP.

**Non-traditional data.

***Authors still used "TOD” but they actually talked about TSA.

****The authors tend to treat both TSAF and TSAP as "TOD-ness".

\# Recommended for the future studies.

Based on Table 1, we can see that not all authors have the same understandings or definitions of TSAF and TSAP. When they started formulating indicators for TSAF and TSAP, this becomes clearer. Singh et al. $(2014,2017)$ treated both TSAF and TSAP as "TOD-ness" and did not differentiate TSAF and TSAP indicators. Similarly, NCHRP (2005), Renee (2007) and Lyu et al. (2016) employed various TSAF and TSAP indicators to measure the TOD performance. Other authors such as Curtis (2012) and Loo and du Verle (2017) regarded employment density as a TSAF contributing to TSAP instead of as a TSAP indicator. The above indicate the complex relationship between TSAF and TSAP. A TSA with few TOD features such as walking/cycling facilities may still enjoy good TSAP, for instance, attracting a lot of park-and-ride transit riders. Similarly, this TSA can still attract a significant number of residents and employers. When more TOD features are added to this TSA, such positive effects as extra residents and employers can gradually entail. Most authors of the literature listed in Table 1 also implicitly or explicitly believe so - that was also why they studied TSAs or advocated TODs (e.g., see Cervero et al., 2017; Dittmar \& Ohland, 2004; Ratner \& Goetz, 2013).

Of all the indicators for TSAF and TSAP listed in Table 1, few can be readily transferred and compared across cities/contexts. This is because most authors still relied on data they could conveniently obtain from different local and/or traditional sources when formulating and/or calculating various indicators. They rarely considered how sources, format and quality of the data can influence the transferability, reliability and validity of their indicators. If we assume, however, all the data issues did not exist, Lyu et al. (2016), Papa and Bertolini (2015), Renee (2007), Singh et al. (2014, 2017) and TCRP (2007) have come up with a comprehensive suite of indicators/indices for TSAF and/or TSAP. Based on responses from different stakeholders and existing practices (e.g., see Caltrans, 2002), NHCRP (2005) also had recommended different sets of indicators/indices for various stakeholders to measure and monitor the TOD success. These indicators/indices can be further customized for local contexts too (e.g., Renee, 2007; Lyu et al., 2016).

\subsection{TSAF and TSAP indicators and NTD}

Compared to traditional data, NTD have such new characteristics as:

- Volume--a much larger size of data;

- Velocity--be generated in or near real-time;

- Variety--have structured, unstructured, temporal and spatial information;

- Exclusive in scope--capture a much larger sample size;

- Higher resolution--hold much more details about the subjects;

- Relational--have much more common fields that diverse datasets can be joined together;

- Flexible and scalable--new fields can be added and size can be expanded efficiently (Kitchin, 2013; Mayer-Schonberger \& Cukier, 2013; McKinsey Global Institute, 2015). 
Of the existing studies we collected and reviewed, only Sung and Oh (2011), Papa and Bertolini (2015) and Lyu et al. (2016) have utilized NTD together with traditional data to help measure TSAF and TSAP. Other than that, little has been done in the existing studies in terms of formulating TSAP and TSAF indicators based on NTD, alone, or its combination with traditional data.

\subsection{Good TSAF and TSAP indicators}

Based on Table 1, one can see that good TSAF/TSAP indicators vary across the literature. Factors such as the purpose of the study, sources or availability of input data, types/categories of TSA and specific feature and/or performance (or dimensions of them) that one wants to consider/emphasize all influence how "good" a TSAF/TSAP indicator is. The introduction of NTD into the formulation of TSAF/TSAP indicators can bring changes regarding how we should define and recommend a "good" TSAF/TSAP indicator. Sung and Oh (2011), for instance, have used smartcard data to derive transit ridership of different TSAs. Such data, in theory, can cover all TSAs and can be segmented into more time intervals and be collected continuously. Thus, if transit ridership is used as an indicator for TSAP, it can/should be measured in a comprehensive and continued way. Similarly, Lyu et al. (2016) have employed Point of Interest (POI) data to derive such indicators as the degrees of functional mix and land-use mix. The POI data is compiled by Baidu, one of the most dominant Internet-service companies in China, whose counterpart outside China is Google. It shows not only the primary land use of different parcels of land (e.g., parking and school) but also the main function of different buildings or complexes (e.g., a restaurant and a shopping mall). Unlike their traditional counterparts (e.g., an official land-use map) that are only updated and published once or twice a year, the POI data is monitored and updated continuously. Thus, any TSAF indicators based on the POI data can in theory be updated continuously too.

\section{$4 \quad$ Empirical studies}

Thanks to the introduction of NTD into TSA studies, we can expect at least two significant changes in the TSAF/TSAP indicators. One is the emergence of new indicators that are purely based on NTD, which provides new information that traditional data do not provide, e.g., real-time locations of transit riders via smartphones; the other is enhanced or revised existing indicators because NTD has generated new/better input data for those indicators, e.g., transit users by more time intervals across an hour. In one manuscript, we cannot enumerate all the new, revised and enhanced TSA indicators because of the advent of NTD. Thus, we here only illustrate several new, revised and enhanced TSAF/TSAP indicators based on NTD in this exploratory/illustrative study.

\subsection{Indicators based on NTD}

The TSA-level indicators we formulated and illustrated were Weibo check-ins, the number of POIs, the number of the POIs with at least one Weibo check-in, the Simpson index of different POIs by main function, and the population based on Baidu heatmaps. In Mainland China, such social media apps as Facebook and Twitter are blocked. Thus, Weibo was developed as the local alternative. It is one of the most popular social media apps in Mainland China now. Like Facebook and Twitter, Weibo generates such information as the location of a user and her/his thoughts on something. In addition, like Google, Weibo and its contractors compile and keep updating various POIs and provide their respective longitude and latitude information.

We acknowledge the complex relationship between TSAF and TSAP. In our exploratory/illustrative study of Shenzhen, we simply used the number of POIs (especially POIs with at least one Weibo check-in, which indicates there was at least one visit) and the Simpson index of different POIs by main function to measure TSAF. We used the population per hour on weekdays or weekends derived from the Baidu heatmaps to measure TSAP. It was our belief that the former could somehow represent how 
many popular destinations and how mixed they are in a TSA whereas the latter indicates how many people (including a significant number of the TSA residents and/or transit riders into the TSA) per hour across a day there are in a TSA. Our indicators thus are comparable to those by Cervero and Kockelman (1997), NCHRP (2005), Singh et al. (2017) and Loo and Du Verle (2017), albeit that our input data for indicators is NTD.

\subsection{The site}

The site for our empirical studies is Shenzhen, which is one of the most successful cities in China's opening-up and reform efforts since 1978. It was a small fishing village until the early 1980s. But it now boasts of a population of 11.9 million and an administrative area of 1,997 square kilometers (SSB \& NBSSOS, 2017). It is thus often called a miracle. Not surprisingly, most areas in Shenzhen were masterplanned and recently developed. Various popular and state-of-the-art planning and development concepts in the world such as TOD and "Property + Rail" were piloted or implemented here, often with much less resistance or objection than those long-established cities. Shenzhen was selected as a pilot city for the "transit metropolis" program in China. As the pilot city, Shenzhen has undertaken many efforts to improve TLUI and has achieved notable accomplishments since 2010 (Shenzhen Committee for Transport and Communication, 2013). According to the latest plan approved by the national government, Shenzhen would eventually have as many as 16 metro lines with a length of 596.9 kilometers (NDRC, 2017). As of August 2017, Shenzhen already had 167 stations on eight metro lines with a total length of 285 kilometers. These stations were located in a wide array of subareas in the city such as downtown, free-trade zone, village-in-the-city and areas outside or inside the Shenzhen Special Economic Zone's internal boundaries ${ }^{1}$, which constitute different backdrops or milieus for the design, construction and operation of various TSAs. As one of the major high-tech hotbeds of China, Shenzhen has a high smartphone penetration rate among its residents. This generates many useful and reliable forms of NTD. Chinese IT giants such as Baidu, Sina and Tencent would never forget about demonstrating and even testing/developing their latest products and services in Shenzhen. Interesting NTD such as on-line Baidu heatmaps and Weibo POIs emerge consequently. In our empirical/illustrative studies of Shenzhen, we thus in theory had various options of NTD. But in practice, we only focused on the options (data) that we could more conveniently have access to. Below were detailed descriptions of those data.

\subsection{Data}

There are two primary sources of NTD used in our empirical studies. One is the Weibo POIs and the other is the Baidu heatmaps. Both were crawled via Application Programming Interface (API) from Weibo and Baidu, respectively. For the Weibo POIs, we used the latest (2017) version (to measure TSAF) and the 2014 version (for comparison purposes). For the Baidu heatmaps, we employed two weekdays (May 17 and 19, 2017) and two weekends (May 20 and 21, 2017) to quantify TSAP. Shenzhen's metro system is "rapid transit" according to American Public Transportation Association (APTA) (APTA, 2009), which operates in dedicated right-of-way with grade separation and involves heavy investment. APTA (2009) considers "primary catchment area" of rapid transit as areas within $2 / 3$ miles (approximately 1,000 meters) of a station. Our TSAs for Shenzhen in this study were thus defined as areas within 1,000 meters of a metro stop.

\footnotetext{
${ }^{1}$ Between 1985 and 2003, Shenzhen used to divide its administrative areas into two portions. For the portion closer to Hong Kong, non-Shenzhen registered residents must apply for a permit to get in, which is called "inside the Shenzhen Special Economic Zone's internal boundaries” (“关内”) . The other portion was called ”areas outside the Shenzhen Special Economic Zone's internal boundaries (“关外”) .
} 
Figure 2 below shows how the TSA-level NTD was processed and used in this study.

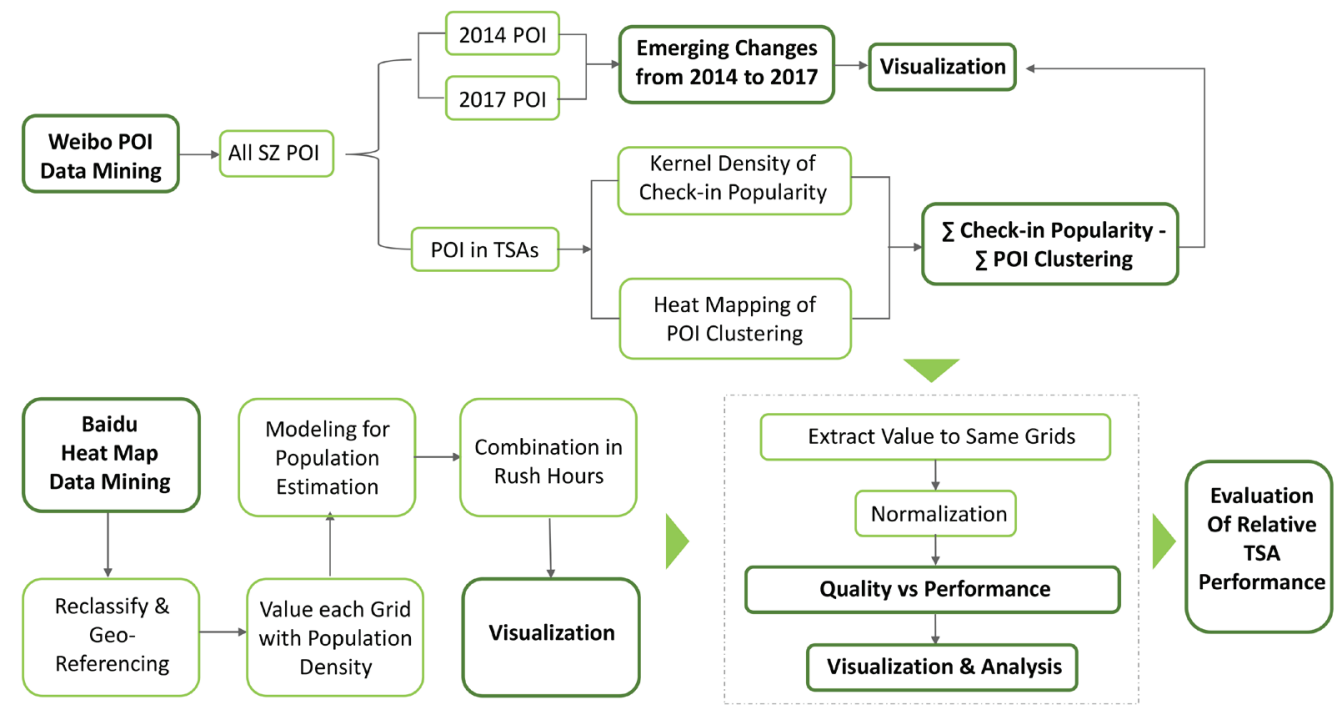

Figure 2: Flowchart of data processing and analysis

In three years, POIs in the 167 TSAs in Shenzhen has more than tripled. In 2014, there were 18,623 POIs of 190 categories. In 2017, the corresponding figures became 56,272 and 500, respectively. Why was there so much growth? It was likely that Weibo and its contractors had compiled and captured more POIs in the three years and had introduced more (sub)categories of POIs as more and new businesses emerged. Most new POIs concentrated in a relatively small number of the TSAs. If we rank all the TSAs by the number of new POIs between 2014 and 2017, for instance, the Top 5, 10, and 20 TSAs accounted for 26, 47 and 81 percent of the all the new POIs, respectively. Most of these top TSAs were inside three older districts of Shenzhen: Luohu, Futian and Nanshan (Figure 3).

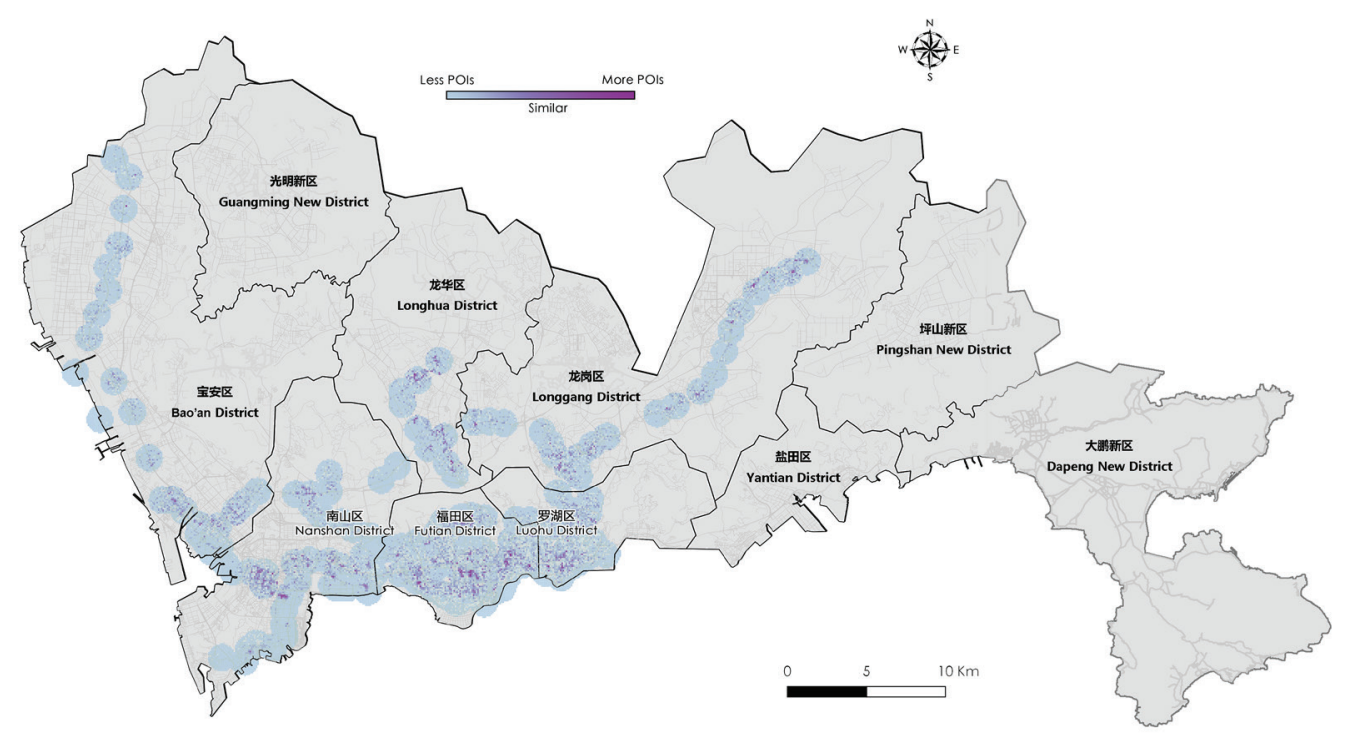

Figure 3: The POI growth by TSAs (January 2014-April 2017) 
Interestingly, the number of POIs is not a sufficient condition for the Weibo check-ins. For the top 20 TSAs with the most Weibo check-ins (January 2014 to April 2017), the correlation coefficient of the Weibo check-ins and the number of POIs (as of April 2017) is only 0.19.

To check whether the mix of different POIs by main function is corrected to the active Baidu app users (derived from Baidu heatmaps, called "the Baidu user" hereafter) at the TSA level, we calculated the Simpson diversity index of all the POIs by main function. Then we estimated the correlation coefficient between this index and the Baidu users. The Simpson diversity index was calculated as:

$\mathrm{D}_{\mathrm{i}}^{\mathrm{t}}=\sum_{\mathrm{j}=1} \mathrm{Jt}\left(\mathrm{p}_{\mathrm{i}, \mathrm{j}}^{\mathrm{t}}\right)^{2}$

where,

$\mathrm{D}_{\mathrm{i}}^{\mathrm{t}}$ is the Simpson diversity index for TSA $\mathrm{i}$;

$\mathrm{J}^{\mathrm{t}}$ is the total types of POI main functions for TSA i;

$\mathrm{p}_{\mathrm{i}, \mathrm{j}}{ }^{\mathrm{t}}$ is the proportion of the $\mathrm{j}$-type POIs in TSA i such that $\sum_{\mathrm{j}=1}{ }^{\mathrm{Jt}} \mathrm{p}_{\mathrm{i}, \mathrm{j}}{ }^{\mathrm{t}}=1$.

In ecology, the Simpson diversity index was initially proposed by Simpson (1949), which measures species diversity within a habitat. In economics, its peer is Herfindahl-Hirschman Index, which measures the market concentration or the level of competition of companies within a market or industry (Herfindahl, 1950; Hirschman, 1945). Somewhat to our surprise, the correlation coefficient of between the Simpson diversity index and the Baidu users is only 0.02. In other words, there are only weak connections between the mix of POIs by main function and the Baidu users.

Nevertheless, the Weibo check-ins and the growth of POIs between 2014 and 2017 are strongly correlated in the space at least at the district level. Where there are more POIs, there are more POI check-ins as well (Figures 3 and 4). These two phenomena together imply that the new POIs could be more effective in terms of attracting Weibo users. This also implies the power of the market: those POIs that could not attract sufficient people (customers) may gradually be phased out and the market was smart and nimble enough to attract new POIs or consolidate or upgrade old POIs in different TSAs in order to efficiently meet the ever-changing demand therein.

Putting the above facts together, we also felt that there could be at least two types of POIs in a TSA, one may attract more Weibo users than the other. The former thus can better reflect the desired TSAF of relevance to Weibo users or even to the public. If we can further differentiate Weibo users or people into or out of a TSA by mode of travel (metro vs. others), we can then better examine whether certain POIs in a TSA contribute more to TSAP, which can be measured by the percentage of metro usage and the metro ridership of a TSA. 


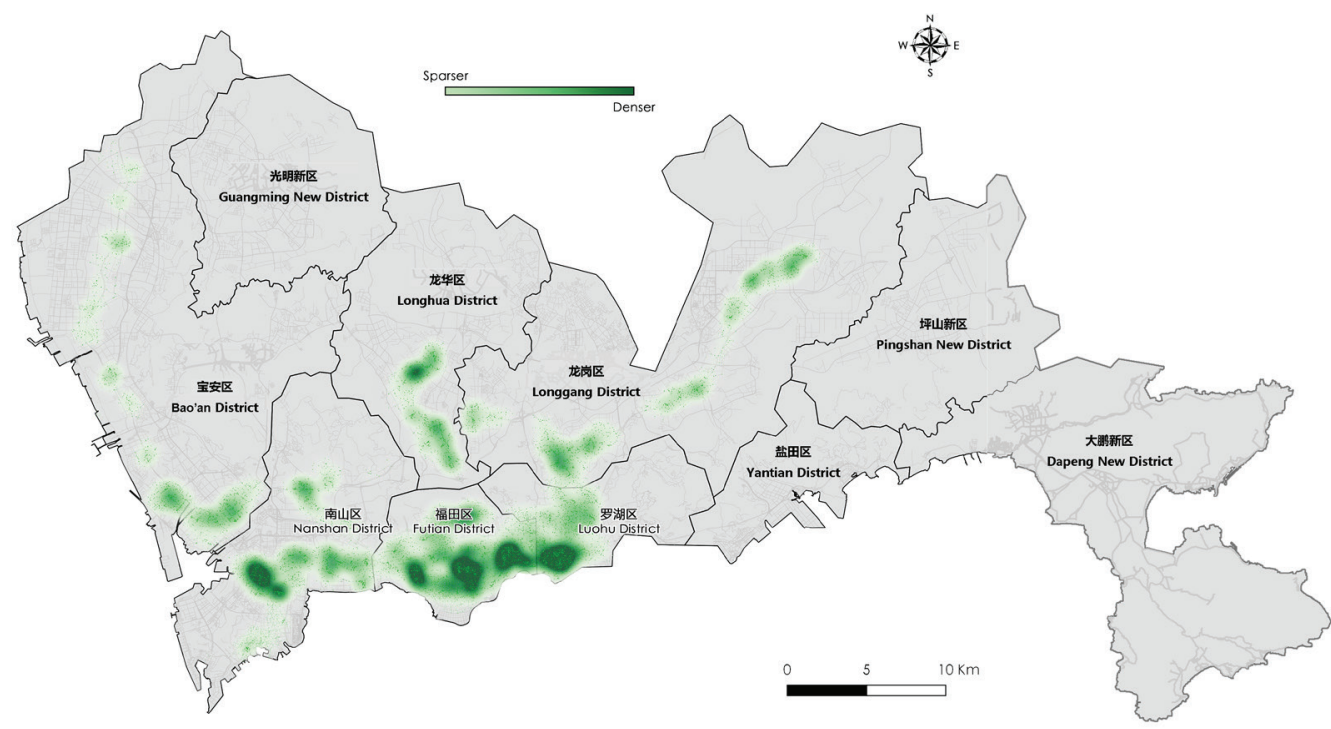

Figure 4: POI check-in heatmap (January 2014-April 2017)

The NTD we had, however, did not allow us to differentiate people into a TSA by mode of travel. But they did allow us to see in addition to Weibo users, on average how many other people there were in a TSA across a day. These people had these characteristics: They had a smartphone and allow Baidu apps and other apps underpinned by Baidu apps to track their real-time spatial location when they used certain "free" services such as Baidu Map and Dianping. Given that Baidu apps (especially Baidu maps and Baidu search) are so popular among smartphone users in Mainland China, it can be expected that Baidu captures the real-time location of a very large percentage of smartphone users therein. The Baidu heatmaps we crawled were based on the captured real-time locations of millions of smartphone users. But Baidu has never released the know-how regarding how it captures those locations, how many and how much percentage of smartphone users are tracked. However, given the above-mentioned facts about Baidu apps, we expected that the Baidu heatmaps captured virtually almost every smartphone user in Mainland China.

We crawled four days (two weekdays and two weekends) of the Baidu heatmaps. A typical Baidu heatmap for Shenzhen is shown in Figure 5. The map changes every hour and so one day can have as many as 24 maps. The maps' different colors represent different density of active Baidu users. Baidu heatmaps were originally in .jpeg format and thus did not directly offer the exact number of active Baidu users by TSA. We vectorized and georeferenced these maps and estimated the number of active Baidu users by TSA, taking advantage of a series of geoprocessing tools or procedures such as "create signature" and "maximum likelihood classification". In a nutshell, these tools and procedures enable us to divide the heatmaps into $100^{*} 100$-meter grids and assign a distinct color, which represents different density of active Baidu users, to each grid. By summing up the Baidu users of all the grids within a TSA, we were able to estimate how many active Baidu users there were. If we assume the number of these users is positively correlated to the TSA population, then those colors also indicate the density of the residential population of different TSAs and whether the density changes between days (e.g., Wednesday vs. Friday and weekdays vs weekends). Such density based on traditional data at a fixed time point has been used by such authors as Cervero and Kockelman (1997), Loo and du Verle (2017) and NCHRP (2005) to 
measure the performance of TODs/TSAs. The density based on the Baidu heatmaps, compared to that based on traditional data, has at least two advantages: First, given that the Baidu heatmaps are available at very low costs, the density can be calculated rather inexpensively and for many TSAs simultaneously; Second, as the Baidu heatmaps are updated constantly, the density can also be updated constantly. However, as we cannot differentiate mode of travel of those active Baidu users simply based on Baidu heatmaps, we still need other data if we hope to measure TSAP in such aspects as transit ridership, car usage and walking behaviors. Similarly, because we cannot know exactly who those active Baidu users were (e.g., residents of a TSA vs. commuters into a TSA), we might also misinterpret, overestimate or underestimate the usage of POIs in a TSA. A commuter into a TSA, for instance, may rarely patronize such destinations as wet market and kindergarten therein.

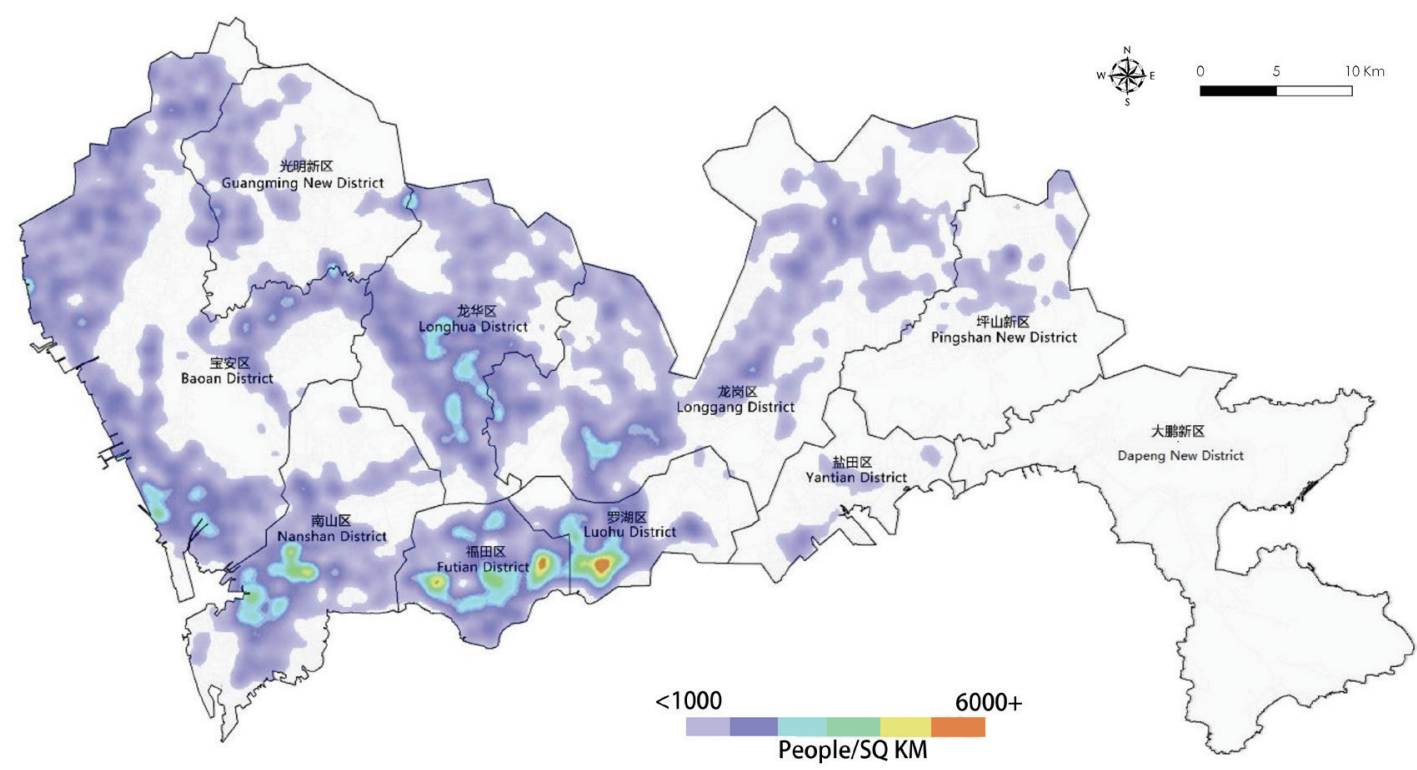

Figure 5: A typical baidu heatmap for Shenzhen

\subsection{Measuring/comparing all the TSAs with NTD-based indicators}

Bearing in mind that some POIs are more popular than others in our daily lives, we used the POIs with Weibo check-ins rather than all POIs as a TSAF indicator. Doing this prevented us from using those obsolete POIs that few would visit. Ideally, we should differentiate the Baidu users by TSA and by mode of travel before using them as a benchmark for TSAP. But Baidu hoards the know-how regarding how it tracks and estimates the Baidu users, thus we had no way to do the differentiation effectively. In the following analyses, we use the Baidu users as a proxy of metro riders and residents in a TSA to measure the TSAP.

Figures 6 and 7 show the relationship between the number of POIs by TSA and the average number of the Baidu users per hour by TSA ("the number of users" as shorthand hereafter) on a weekday (May 17, 2017) and on a weekend (May 20, 2017). To better visualize the relationship, we first divide TSAs into four clusters by the number of users. Then in both Figures 6 and 7, we ensure that the size of the circles is proportional to the number of users across the clusters. 


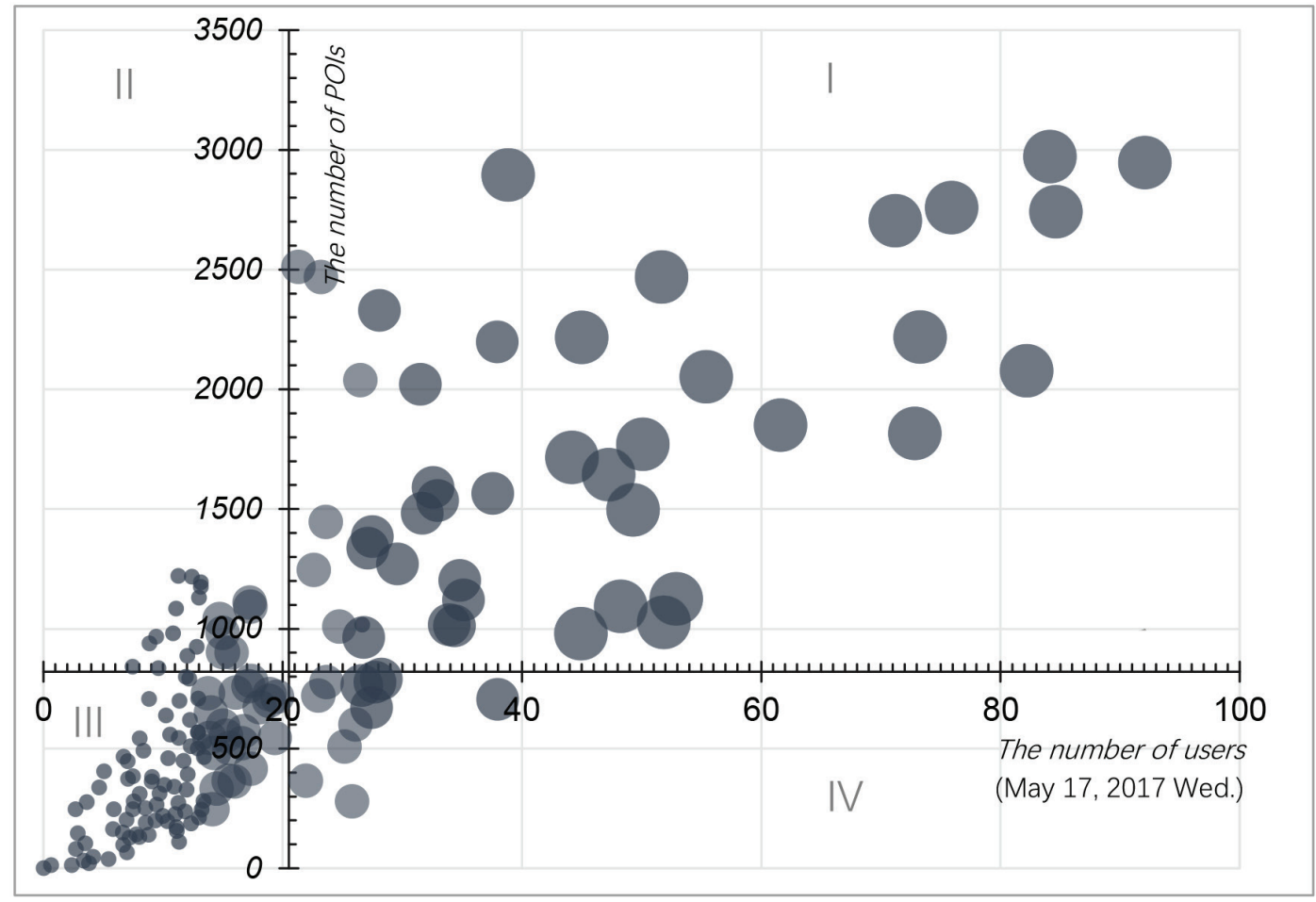

Figure 6: All TSAs' POIs and the number of users on a weekday

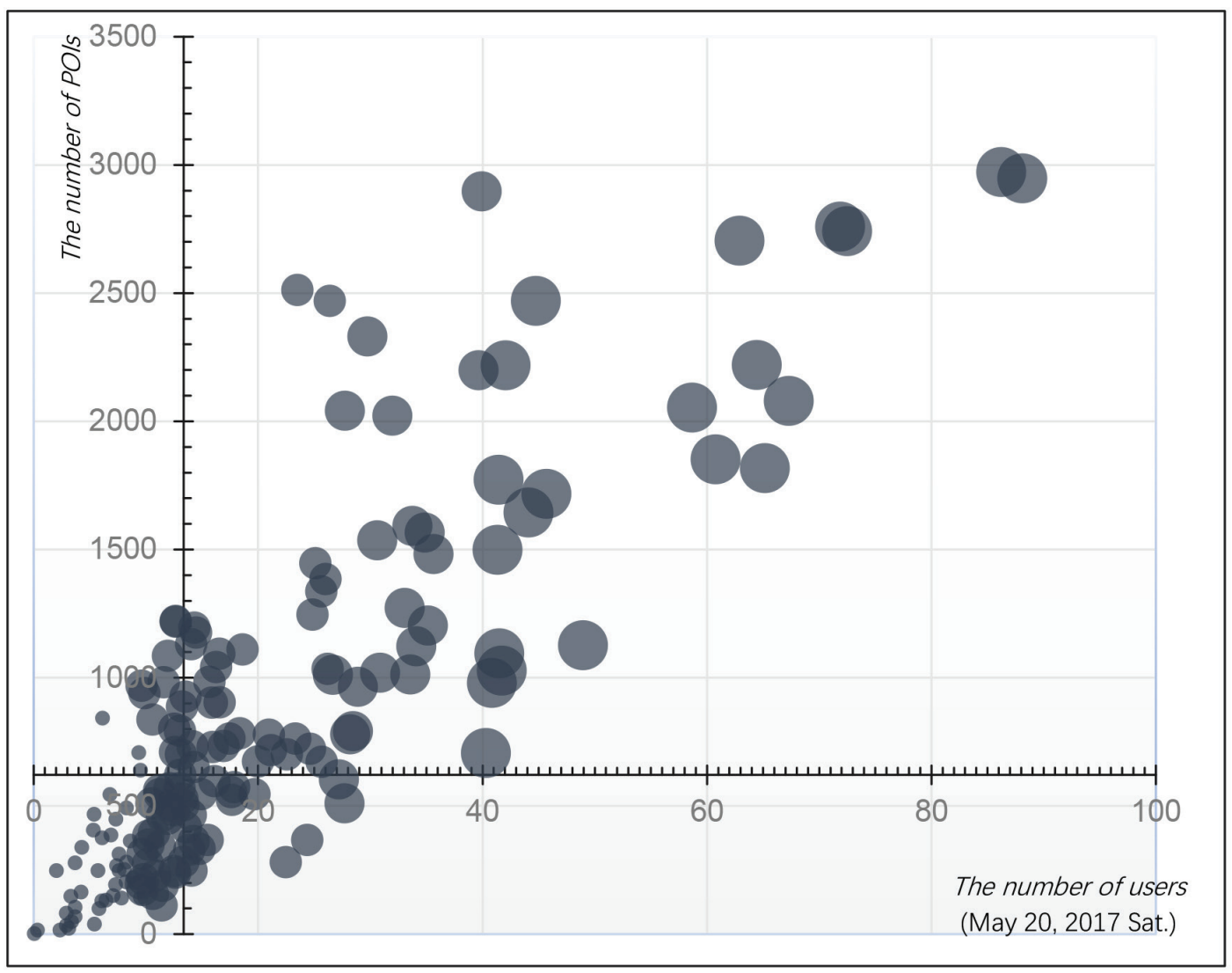

Figure 7: All TSAs' POIs and the number of users on a weekend 
We set the origin of Figures 6 and 7 as the means of the numbers of POIs and users. As such, the 167 TSAs were classified into four quadrants (See Figure 6 for designations of the quadrants). In Quadrant I, all the TSAs herein have more POIs and users than the mean. In Quadrant II, all the TSAs herein have more POIs than the mean but fewer users than the mean. In Quadrant III, all the TSAs herein have fewer users and POIs than the mean. In Quadrant IV, all the TSAs herein have fewer POIs than the mean but more users than the mean.

Assuming each POI consumes the same resource to create and resource saving and economic efficiency is our primary goal, Quadrant IV TSAs are the most desirable from the resource-saving perspective. In the same vein, Quadrant II TSAs are the least desirable where there is an "oversupply" of POIs (or in other words, there are some inefficient POIs). For Quadrants I and III TSAs, there is match between the numbers of POIs and users. There are more/fewer POIs but there are also more/fewer users. We can regard all these TSAs as "performing" ones. For all TSAs across four quadrants, there is a strong positive correlation between the numbers of POIs and users (correlation coefficient is 0.69). This may again reflect the power of the market. There was a "co-location" between POIs and users (c.f., Levinson, 1998).

Between different TSAs within one quadrant, however, there are still notable differences in terms of the POI efficiency: how many users one POI on average can possibly "generate". For Laojie and Guomao, two TSAs in Quadrant I, for example, Guomao's POIs were on average more efficient on weekends. We can use the POI efficiency to further differentiate TSAs in the four quadrants, respectively. For instance, for all Quadrant II TSAs, even though all of them were undesirable, we can still find the POI efficiency's median. Using this median as a benchmark, we can see that some TSAs still outperform others. In other words, based on NTD such as Weibo check-ins and Baidu heatmaps, we could find useful indicators to measure/compare the features and performance of different TSAs. More importantly, by employing NTD for different periods (e.g., weekdays vs. weekends and AM hours vs PM hours) and for different TSAs, we are able to continuously or strategically monitor TSAF and TSAP and their respective changes. In the case of Shenzhen, for instance, not all TSAs equally perform on weekdays and weekends. If we want to create some TSAs perform well on both weekdays and weekends, then NTDbased indicators such as the numbers of POIs and users would be helpful. We can, for instance, introduce more POIs that enjoy high efficiency on both weekends and weekdays into the TSAs in question.

\subsection{Underlying reasons for a TSA fell into a specific quadrant}

Why did a TSA fall into a specific quadrant but not the others? We conducted an interview-based study of four TSAs to probe into the reasons. The interviewees of our study were three local planners who grew up and continuously practiced in Shenzhen. The four TSAs were Hubei, Conference and Exhibition Center (CEC), Shekou Port and Ludancun. They are a Quadrant I, II, III and IV TSA on both weekdays and weekends, respectively.

Based on the opinions of the local planners, Hubei has very diverse land uses, building types/ages, employment opportunities and populations. It is one of the oldest neighborhoods in the city. Diversity and history made Hubei attractive to a lot of people and activities. Thus, when we used the numbers of users and POIs to represent those people and activities, Hubei was a Quadrant ITSA that has many people and many POIs simultaneously.

$\mathrm{CEC}$ was a recent hotspot for public and private investment. The construction of $\mathrm{CEC}$ was led and funded by Shenzhen Municipal Government (SMG). Having strong confidence in SMG, many concurrent or subsequent multifunctional buildings/complexes funded by the private sector have clustered in/around CEC. On the one hand, these buildings/complexes aimed to serve not only local residents or workers inside CEC but also residents, tourists or consumers outside CEC and thus were built in a 
comparatively large quantity; on the other hand, most of these buildings/complexes cater to high-end customers and often have little to do with average people. There is a notable oversupply of POIs from the perspective of average people: at night and during weekends, most daytime/weekday workers in CEC stayed at home; during weekdays, except workers or residents in CEC, few patronized the POIs therein. Eventually, CEC became a Quadrant II TSA, especially during weekday nights and on weekends.

Shekou Port is an interesting place, which is on a peninsula that is far away from the two CBDs in Shenzhen: Luhu and Futian. It used to be a busy seaport for both goods and people. Administratively, it was not part of the City of Shenzhen until 1992. China Merchants Group (CMG) used to own and operate Shekou Port. CMG set up its own planning and construction systems for the port and its vicinities, where understandably most developments were led or financed by CMG. In recent years, the port and its vicinities ("Shekou" for shorthand hereafter) have experienced great transformations because of the establishment of Qianhai Free Trade Zone (QFTZ). QFTZ had a goal to attract more investors from Hong Kong, Macau and beyond. Shekou was included as a part of QFTZ. A modern cruise terminal and some high-end residential estates and business/office buildings had emerged thanks to QFTZ. But as a whole, those developments were only attractive to a small portion of people, businesses and employers. Thus, compared to Hubei and Ludancun, Shekou was a still place (Quadrant III TSA) where there were fewer POIs and fewer residents/visitors.

Similar to Hubei, Ludancun also has a long history. But its land-use mix is not as diverse as Hubei. Its commercial opportunities and residential options, for instance, are dominated by big-box complexes and employer-subsidized Danwei compounds, respectively. Those complexes and compounds are not big in quantity; however, they still attracted a significant number of incoming commuters, residents and visitors. In other words, each complex (or compound) is an efficient POI. This made Lundancun a Quadrant IVTSA.

\section{$5 \quad$ Conclusions and discussion}

We can never over-exaggerate the roles of transit and TLUI in our battle against the rising popularity of private car usage, increased traffic congestion on roads and worsening ambient air quality in big cities. TSAs are a crucial interface between transit services and residents. They have also become increasingly desirable spaces in our cities. The quality of such interface profoundly influences the attractiveness of transit services and the sense, charm and identity of a place/space. TODs, a subset of TSAs, have been extensively studied before because of that. But till today, there has not been an agreement about how to measure the success/performance of TODs/TSAs and how to use some indicators/benchmarks to differentiate various TSAs (Hale, 2014). In this study, we first show that it is partially because of the characteristics of input data that led to the above situations. In most existing TSA/TOD studies, the input data has been traditional data. We then argue that the advent of NTD can help improve the situations. NTD can be used to enhance and supplement many TSAF and TSAP indicators. Our case study of Shenzhen, for instance, shows that emerging POI data can supplement and even replace traditional land-use maps to quantify land-use intensity and diversity of TSAs. In addition, the Weibo check-ins and Baidu heatmaps also enable us to fathom how popular a TSA is among local residents more conveniently and efficiently than traditional data.

Based on the case of Shenzhen, we can have the following findings and/or lessons, some of which might be transferrable to other locales as well:

First, it is feasible to measure and even continuously monitor features and performance of all important TSAs (e.g., all TSAs around major metro stations) based on NTD. In this study, for instance, we illustrate that TSAF could be measured by the number and the mix of POIs and TSAP could be measured by the number of active Baidu users. 
Second, the new TSAF and TSAP indicators based on NTD, alone, or its combination with traditional data could generate new knowledge about TSAs. In Shenzhen, we used the active Baidu users to show how TSAP varied across locales and turned to the local experts for us to understand what caused the variances. The Guomao TSA, for instance, has approximately 12,000 more active Baidu users and 1,700 more POIs with Weibo check-ins than the Airport E. TSA on a typical weekday. Even for the same TSAs, their TSAP can vary across days. The extreme TSAs in all the four quadrants can be totally different across days (See Figures 6 and 7). The new TSAF and TSAP indicators based on NTD also enable us to reflect upon findings and conclusions in the existing studies. We found, for instance, a good POI mixture (measured by Simpson diversity index) might not generate a large flow of people (measured by the number of users) into a TSA. This means that certain POIs can contribute more to flows of people into TSAs.

Third, the POI efficiency could be a useful indicator for us to differentiate/rank TSAP within a city or across cities. Measuring longitudinal and cross-sectional changes in the POI efficiency enables us to see not only whether one TSA outperforms than others in the same period but also whether this TSA has performed better (or more poorly) now than before, within a city or across cities. It also partially reflects the dynamics and vicissitude of TSAs and even their respective host cities or regions.

Fourth, our case studies of the four TSAs in Shenzhen indicate that traditional data and NTD can mutually supplement each other in TSA studies. By combing traditional data and NTD, we were able to first efficiently categorize a large number of TSAs in the city based on NTD and some predefined criteria. Then we used such traditional data as interviews to understand better why extreme TSAs (see Figures 6 and 7) performed or underperformed. The interviews provided extra knowledge and information about local history, public policies and plans that influenced TSAP and TSAF that NTD alone could not. The case studies have also generated some interesting insights into the factors contributing to TSAP and TSAF across locales. They showed that history matters and governmental interventions might not be inefficient in terms of promoting TSAP and/or enhancing TSAF. Both Hubei and Ludancun, for instance, enjoy a long history but experienced relatively fewer governmental interventions. Here, the market played a larger role as to determine what POIs should be there and how many. This led to efficient POIs that attract constant people/customer flows. In contrast, both CEC and Shekou Port were primarily outcomes of large-scale and continuous governmental interventions. The interventions were impactful in terms of producing POIs that efficiently attracted high-end employers (businesses) and customers. But they might have "gentrified" POIs that catered to other employers and customers. As a result, the POIs in CEC and Shekou Port were not able to generate constant flows of people across hours of a day or across days of a week. As a whole, the four cases indicate that to better promote TSAP and/or enhance TSAF, decision-makers should treasure and harness history and carefully balance the market and the governmental intervention.

Despite the above findings and/or progresses, our studies can be further improved in the future, at least in the following aspects. First, introduction of other NTD to measure the TSAF and TSAP more comprehensively and accurately. One important TSAF, for instance, is about human scale, we could use geotagged photos or comments in Google Map to help measure it. In addition, given that transit usage is a better indicator for the TSAP, we could use smartcard swipes to better estimate transit riders into TSAs. Second, given that TSAF and TSAP are multidimensional, we should consider some indices instead of indicators. Both traditional data and NTD can be input for these indices. We can, for instance, emulate ITDP (2017) and Lyu et al. (2016) to rank TSAP and TSAP based on expert opinions. Third, we need to conduct more case studies to understand better the complex relationship between TSAF and TSAP. Our current studies, for instance, already show that not all POIs contribute to the popularity of TSAs among the active Baidu users. In those case studies, we should further examine which types 
of POIs or combination of POIs contribute the most to TSAP. Last but not least, we have treated the level and carrying capacity of transit services as exogenous when examining TSAF and TSAP. It would be interesting to better account for transit service in our studies of TSAs. We could, for instance, have different TSAP benchmarks for TSAs served by different types and/or levels of transit services. 


\section{References}

American Public Transportation Association (APTA). (2009). Defining transit areas of influence. Washington, DC: APTA.

Arrington, G. B., \& Parker, T. (2001). Factors for success in California's transit-oriented development. Sacramento, CA: California Department of Transportation, Statewide Transit-Oriented Development Study.

Batty, M. (2013). Big data, smart cities and city planning. Dialogues in Human Geography, 3(3), 274 279.

Belzer, D., \& Autler, G. (2002). Countering sprawl with transit-oriented development. Issues in Science and Technology, 19(1), 51-58.

Bernick, M., \& Cervero, R. (1997). Transit villages for the 21st century. New York: McGraw-Hill.

Boarnet, M., \& Crane, R. (1998). L. A. story: A reality check for transit-based housing. Journal of the American Planning Association, 63(2), 189-204.

Bertolini, L. (1996). Nodes and places: Complexities of railway station redevelopment. European Planning Studies, 4(3), 331-345.

Bertolini, L. (1999). Spatial development patterns and public transport: The application of an analytical model in the Netherlands. Planning Practice and Research, 14(2), 199-210.

Bertolini, L., Curtis, C., \& Renee, J. (2012). Station area projects in Europe and beyond: Toward transit oriented development? Built Environment, 38(1), 31-50.

Bukowski, B., Boatman, D., Ramierz, K., \& Du, M. (2013). A comparative study of transit-oriented development in Hong Kong. Retrieved from https://goo.gl/rL19Gp

California Department of Transportation (Caltrans). (2002). Statewide transit-oriented development study: Factors for success in California. Sacramento, CA: Caltrans.

Calthorpe, P. (1993). The next American metropolis: Ecology, community, and the American dream. Princeton, NJ: Princeton Architectural Press.

Carlton, I. (2007). Histories of transit-oriented development: Perspectives on the development of TOD concept (WP-2009-02). Berkeley, CA: Institute of Urban and Regional Development, University of California, Berkeley.

Cervero, R. Guerra, E., \& Al, S. (2017). Beyond mobility: Planning cities for people and places. Washington, DC: Island Press.

Cervero, R., \& Kockelman, K. (1997). Travel demand and the 3Ds: Density, diversity, and design. Transportation Research D, 2(3), 199-219.

Cervero, R., Ferrell, C., \& Murphy, S. (2002). Transit Cooperative Research Program, Research results digest Number 52. Washington, DC: Transportation Research Board, National Research Council.

Cervero, R. (1998). Transit metropolis: A global inquiry. Washington, DC: Island Press.

Cevero, R., \& Murakami, J. (2009). Rail and property development in Hong Kong: Experiences and extensions. Urban Studies 46(10), 2019-2043.

Cevero, R., Murphy, S., Ferrell, C., Goguts, N., Tsai, Y., Arrington, G.B., .. \& W Witenstein, N. (2004). Transit-oriented development in the United States: Experiences, challenges, and prospects. Washington, DC: Federal Transit Administration.

Curtis, C. (2012). Transitioning to transit-oriented development: The case of Perth, Western Australia. Urban Policy and Research, 30(3), 275-292.

Dill, J. (2008). Transit use at transit-oriented developments in Portland, Oregon, Area. Transportation Research Record, 2083, 159-167.

Dittmar, H., \& Ohland, G. (2004). The new transit town: Best practices in transit-oriented development. Washington, DC: Island Press. 
Dong, H. (2017). Transit induced neighborhood change and the affordability paradox of TOD. Journal of Transport Geography, 63, 1-10.

DT Caijing. (2017). Rediscover Shenzhen on the rail 2017: Shenzhen urban big data vitality report. Shanghai: DT Caijing.

Ewing, R., \& Cervero, R. (2001). Travel and the built environment-synthesis. Transportation Research Record, 1780, 87-114.

Frank, L. D., Saelens, B. E., Powell, K. E., \& Chapman, J. E. (2007). Stepping toward causation: Do built environments or neighborhood and travel preferences explain physical activity, driving, and obesity? Social Science \& Medicine, 65(9), 1898-1914.

Griffiths, B, \& Curtis, C. (2017). Effectiveness of transit oriented development in reducing car use: Case Study of Subiaco, Western Australia. Urban Policy and Research, 35, 4, 391-408

Hale, C. (2014). TOD versus TAD: The great debate resolved...(?). Planning, Practice and Research, 29(5), 492-507.

Herfindahl, O. C. (1950). Concentration in the steel industry. New York: Columbia University Press.

Hirschman, A. O. (1945). National power and the structure of foreign trade. Berkeley, CA: University of California Press.

Institute for Transport and Development (ITDP). (2017). TOD standards version 3.0. New York: ITDP.

Jeihani, M., \& Zhang, L. (2013). Development of a framework for transit-oriented development. Annapolis, MA: Maryland Department of Transportation.

Kahn, M. (2007). Gentrification trends in new transit-oriented communities: Evidence from 14 cities that expanded and built rail transit systems. Real Estate Economics, 35, 2, 155-182.

Kamruzzaman, M., Shatu, F. M., Hine, J., \& Turrell, G. (2015). Commuting mode choice in transit oriented development: Disentangling the effects of competitive neighborhoods, travel attitudes, and self-selection. Transport Policy, 42, 187-196.

Kitchin, R. (2013). The data revolution: Big data, open data, data infrastructures and their consequences. London, UK: Sage.

Levinson, D. M. (1998). Accessibility and the journey to work. Journal of Transport Geography, 6(1), $11-21$.

Liu, L., Du, J., \& Zhao, D. (2013). City of rail: Urban and transport research on Tokyo metropolitan area. Beijing: China Communication Press.

Loo, B. P. Y. (2009). Transport, urban. In R. Kitchin \& N. Thrift (Eds.), International encyclopedia of human geography (pp. 465-469). Oxford: Elsevier.

Loo, B. P. Y., \& du Verle, F. (2017). Transit-oriented development in future cities: Toward a two-level sustainable mobility strategy. International Journal of Urban Sciences, 21, 54-67.

Loo, B. P. Y., Chen, C., \& Chan, E. T. H. (2010). Rail-based transit-oriented development: Lessons from New York City and Hong Kong. Landscape and Urban Planning, 97, 202-212.

Lyu, G., Bertolini, L., \& Pfeffer, K. (2016). Developing a TOD typology for Beijing metro station areas. Journal of Transport Geography, 55, 40-50.

Mayer-Schonberger, V., \& Cukier, K. (2013). Big data: A revolution that will transform how we live, work, and think. New York: Houghton Mifflin Publishing House.

McKinsey Global Institute. (2015). Open data: Unlocking innovation and performance with liquid information. Retrieved from http://goo.gl/LavBty

Morris, J. N., \& Hardman, A. E. (1997). Walking to health. Sports Medicine, 23(5), 306-332.

National Development and Reform Committee (NDRC). (2017). Approval of Shenzhen metro construction plan (2017-2020). Beijing, China: NDRC. Retrieved from http://www.ndrc.gov.cn/zctb/ zcfbtz/201707/t20170712_854358.html 
National Cooperative Highway Research Program (NCHRP). (2005). Transit-oriented development: Developing a strategy to measure success. Washington, DC: The National Academies Press.

Nasri, A., \& Zhang, L. (2014). The analysis of transit-oriented development (TOD) in Washington, DC, and Baltimore metropolitan area. Transport Policy, 32, 172-179.

Newman, P., \& Kenworthy, J. (1999). Sustainability and cities: Overcoming automobile dependence. Washington, DC: Island Press.

Norland, R., \& DiPetrillo, S. (2015). Transit-oriented development and the frequency of modal use. Journal of Transport and Land Use, 8(2), 21-44.

Norland, R. B., Ozbay, K., DiPetrillo, S., \& Lyer, S. (2014). Measuring benefits of transit oriented development. San Jose, CA: Mineta National Transit Research Consortium, San Jose State University.

Olaru, D., \& Curtis, C. (2015). Designing TOD precincts: Accessibility and travel patterns. European Journal of Transport and Infrastructure Research, 15(1), 6-26.

Ong, P., Pech, C., \& Ray, R. (2014). TOD Impacts on businesses in four Asian American neighborhoods. Los Angeles: UCLA Center for the Study of Inequality.

Papa, E., \& Bertolini, L. (2015). Accessibility and transit-oriented development in European metropolitan areas. Journal of Transport Geography, 47, 70-83.

Reades, J. E., Zhong, C., Manley, E., Milton, R., \& Batty, M. (2016). Finding pearls in London's Oysters. Built Environment, 42(3), 365-381.

Ratner, K. A., \& Goetz, A. R. (2013). The reshaping of land use and urban form in Denver through transit-oriented development. Cities, 30, 31-46.

Renee, J. L. (2007). Measuring the performance of transit-oriented developments in Western Australia. Perth, Australia: Institute for Sustainability and Technology Policy, Murdoch University.

Renee, J .L. (2009). Measuring the success of transit oriented development. Perth, Australia: Institute for Sustainability and Technology Policy, Murdoch University.

Renne, J. L., Bartholomew, K., \& Wontor, P. (2011). Transit oriented and joint development: Case studies and legal issues. In Legal Research Digest 36 of Transit Cooperative Research Program. Washington DC: Federal Transit Administration.

Renne, J. L. (2018). Transit-oriented development and ports: A national analysis in the United States. Journal of Transport and Land Use, 11(1), 297-304.

Rodriguez, D. A., \& Vergel-Tovar, C. E. (2018). Urban development around bus rapid transit stops in seven cities in Latin-America. Journal of Urbanism: International Research on Placemaking and Urban Sustainability, 11(2), 175-201.

Shenzhen Committee for Transport and Communication. (2013). Report on Shenzhen's efforts to become an international-level transit metropolis. Shenzhen, China: Shenzhen Committee for Transport and Communication. Retrieved from http://www.transformcn.com/Topics/img/site22/20171204/ c81f661ac8d91b8f33c701.pdf

Shenzhen Statistics Bureau (SSB) \& National Bureau of Statistics' Survey Office in Shenzhen (NBSSOS). (2017). Shenzhen statistical year book. Beijing: China Statistics Press.

Simpson, E. H. (1949). Measurement of diversity. Nature, 163, 688.

Singh, Y. J., Fard, P., Zuidgeest, M., Brussel, \& M. van Maarseveen, M. (2014). Measuring transit oriented development: A spatial multi criteria assessment approach for the City Region Arnhem and Nijmegen. Journal of Transport Geography, 35, 130-143.

Singh, Y. J., Lukman, A., Flacke, J., Zuidgeest, M., \& van Maarseveen, M. (2017). Measuring TOD around transit nodes: Toward TOD policy. Transport Policy, 56, 96-111.

Sung, H., \& Oh, J. T. (2011). Transit-oriented development in a high-density city: Identifying its association with transit ridership in Seoul, Korea. Cities, 28(1), 70-82. 
Suzuki, H., Cervero, R., \& Luchi, K. (2013). Transforming cities with transit. Washington, DC: The World Bank.

Transit Cooperative Research Program (TCRP). (2007). Transit oriented development traveler response to transportation system changes, Report 95. Washington, DC: Federal Transit Administration.

TCRP (2002). Transit-oriented development and joint development in the United States: A literature review. Washington, DC: Federal Transit Administration.

Wang, K., \& Woo, M. (2017). The relationship between transit rich neighborhoods and transit ridership: Evidence from the decentralization of poverty. Applied Geography, 86, 183-196.

Zhao, P., \& Li, X. (2018). Suburbanization, land use of TOD and lifestyle mobility in the suburbs: An examination of passengers' choice to live, shop and entertain in the metro station areas of Beijing. Journal of Transport and Land Use, 11(1), 195-215.

Zhou, J. (2016). Transit metropolis with Chinese characteristics? Literature review, surveys and comparative studies. Transport Policy, 51, 115-125. 